\title{
Reply to Stephany \& Christofidou 2010
}

\author{
Maria Mastropavlou \\ University of Ioannina \\ mariamst@gmail.com
}

First of all, I would like to thank the editors of the Journal of Greek Linguistics for giving me this opportunity, by way of a reply to Stephany \& Christofidou 2010 (this issue) (henceforth, $\mathrm{S} \& \mathrm{C}$ ), to provide some clarifications concerning my initial review of their work (S\&C 2008; see JGL 9 (2009), 215-230), with the aim also of offering a better characterization of my initial arguments.

To begin with, although I do not fully agree, I completely welcome S\&C's argumentation on a usage-based approach to morphological structure and development. I adhere to my initially stated points for consideration and I would only like to point out that S\&C might be doing generative models an injustice by stating that they "assume complex forms to be constructed by adding affixes to stems" (p. 118). It is quite frequently claimed in generative morphology that words are also stored (and therefore accessed and retrieved) as wholes in the lexicon, while word formation rules are used during morphological processing (input analysis) and in generating new words (acknowledging thus the productive nature of morphology) (Selkirk 1982). Although S\&C deny that usage-based models do not recognise processes of morphological decomposition or analysis (as I commented in my initial review), they go on to argue for schemas that account for morphological structure "without however decomposing complex forms into their elements" (p. 118). I do not address the issue of morphological decomposition further at this point, as I think I have provided sufficient detail on my views in the original review.

Regarding the description and analysis of the data provided by the authors in the monograph, I would like to clarify that I was not disappointed by the fact that no well-defined stages of acquisition are described but rather by the fact that no general patterns of acquisition are identified in the language data examined. By general patterns I am definitely not referring to stages but to similar phenomena or types of linguistic behaviour that could be found across the different language samples examined and could very well progress 
gradually rather than in well-defined stages. If there are no patterns in the way children acquire morphology, then one can only describe individual cases independently but cannot talk about acquisition processes for a population in general. However, it could be the case that patterns did actually occur but were not easily identified in the description given in the monograph. In my view, the excessive focus on detail could have made the identification of linguistic patterns difficult.

As far as the methodology is concerned, I fully acknowledge what S\&C state in their response regarding the sample size. I agree that the significance of longitudinal studies does not lie in the size of samples but rather in the description of the way different aspects of language competence emerge in child language. However, my main concern regarding the sample studied here does not so much lie in its size as in the inconsistency regarding the developmental phases investigated. As I stated in the review, not all developmental phases could be investigated for all the children that participated in the study, which might have led to significant information or patterns being overlooked.

Additionally, the authors state in their response that the main findings in their study point to the assumption that "the three grammatical categories of the Greek noun, case, number and gender, develop in parallel ways in the five children studied" (p. 119). I have two concerns regarding this statement. One involves the "parallel" development in the five children, which might have not been the case if data from all developmental phases had been studied for all children. My second concern is that, although S\&C state they investigate grammatical categories, it seems in the data presented that they actually investigate the emergence of specific forms - or distinction between forms - rather than categories. I find the term 'case and number distinctions', which S\&C use rather frequently, to be more accurate.

Regarding the issue of noun classification, S\&C state in their reply that Ralli's work $(2002 ; 2005)$ is not overlooked, but is rather subsumed under the proposal that noun classification depends on the distribution of inflectional endings. However, it is my understanding that Ralli suggests that phonological criteria (mainly what she calls 'inflectional class', IC) constitute only one type of noun classification criteria. She classifies nouns based on IC and gender information (for example, masculine nouns in -os belong to IC1, while neuter nouns in -os in IC7). I see this account as being somewhere between the two accounts described by $S \& C$ (the one arguing for gender criteria and the second arguing for inflectional criteria alone), as she in a sense combines the two. In fact, although Ralli's work is indeed cited at different points in the manuscript, and her views have been described in previous work by 
Christofidou, cited in S\&C 2008, her proposals are not given appropriate attention in this specific manuscript. In my view, it is inconsistent to refer to just two approaches to noun classification.

On the issue of gender determination, $\mathrm{S} \& \mathrm{C}$ state that the review provides an incorrect account of their views. Actually, it is stated in their reply that they "do not doubt that Greek gender assignment is arbitrary ("lexical" in Ralli's terminology), rather than semantically based, as is generally the case in IndoEuropean languages". If that is the case, then the phrase in S\&C 2008: 9, "as is common in Indo-European languages, the Greek gender system comprises a more or less small core of semantic gender assignment based on animacy and sex distinctions", should be rephrased, as it obviously leads to misunderstandings.

Finally, S\&C dispute my initial concern that the role of the underlying nature of the grammatical features investigated has been overlooked. It has been my impression that the underlying differences among the three grammatical features investigated are not taken into account. While gender, on one hand, is considered to be a lexical (or arbitrary) feature of the noun, meaning that a noun is (usually) stored in the lexicon along with a gender value, case, on the other hand, is a syntactic feature in the sense that a noun does not store a case value in its lexical entry, but rather obtains a value (nom - gen - acc) based on its position in a syntactic structure and on syntactic relations. Number, lying somewhere between gender (highly lexical) and case (highly syntactic), differs from both in that it is determined by mainly semantic or pragmatic criteria is also involved in syntactic relations (through agreement). What S\&C seem to overlook is the possibility that these differences might affect the way the three features develop in child language and could (even partly) account for the differences found in the developmental patterns. Of course, I am not suggesting that $S \& C$ should acknowledge this possibility as a fact, but rather that a consideration of its presence, and even argumentation against such a possibility, might lead to a more complete account.

\section{References}

Ralli, Angela. 2002. The role of morphology in gender determination: Evidence from Modern Greek. Linguistics 40: 519-551.

Ralli, Angela. 2005. Morfologia [Morphology]. Athens: Patakis.

Selkirk, Elizabeth. 1982. The syntax of words. Cambridge, MA: MIT Press.

Stephany, Ursula \& Anastasia Christofidou. 2008. The Acquisition of Greek Case, Number, and Gender: A Usage-Based Approach. Allgemeine Sprachwissenschaft, Institut für Linguistik, 
Universität zu Köln, Arbeitspapier Nr. 55. August 2008. ISSN 1615-1496 < http://www .uni-koeln.de/phil-fak/ifl/asw/forschung/ap/StephChrist_AP55.pdf>.

Stephany, Ursula \& Anastasia Christofidou. 2010. Response to Review of The Acquisition of Greek Case, Number, and Gender: A Usage-Based Approach by Maria Mastropavlou. Journal of Greek Linguistics 10(1):117-121. 
